\title{
Síndrome y deformidad de Haglund
}

\author{
Haglund's syndrome and deformity \\ Luis Gerardo Domínguez Gasca, * Luis Gerardo Domínguez Carrillo \\ Citar como: Domínguez GLG, Domínguez CLG. Síndrome y deformidad de Haglund. Acta \\ Med. 2021; 19 (1): 146-147. https://dx.doi.org/10.35366/98592
}

El síndrome de Haglund se caracteriza clínicamente por el engrosamiento de los tejidos blandos retrocalcáneos, existe tendinitis en inserción del tendón de Aquiles, bursitis retrocalcánea y prominencia del borde posterosuperior del calcáneo; fue descrito inicialmente por Patrick Haglund en 1928; el factor predisponente más frecuente es el calzado ajustado; ${ }^{1}$ otros factores asociados son el arco plantar aumentado (pie cavo), un tendón demasiado tenso y cualquier otra situación que propicie la fricción entre el tendón y el hueso.

Por otra parte, la deformidad de Haglund es una alteración en la morfología del calcáneo que forma una prolongación ósea vertical en la tuberosidad posterosuperior. Se han propuesto varios criterios de medida para diagnosticarla, el método más usado es el de las líneas de inclinación paralelas (parallel pitch lines); ${ }^{2}$ recientemente se ha implementado una nueva medición denominada "longitud relativa del calcáneo", que corresponde a la relación X/Y de longitud del calcáneo, siendo X la longitud total del calcáneo, desde el punto más anterior de la apófisis mayor hasta el punto más posterior del calcáneo, donde Y corresponde a la longitud de la tuberosidad mayor, desde el punto más posterior de la superficie del tálamo calcáneo hasta la cumbre de la tuberosidad mayor teniendo como
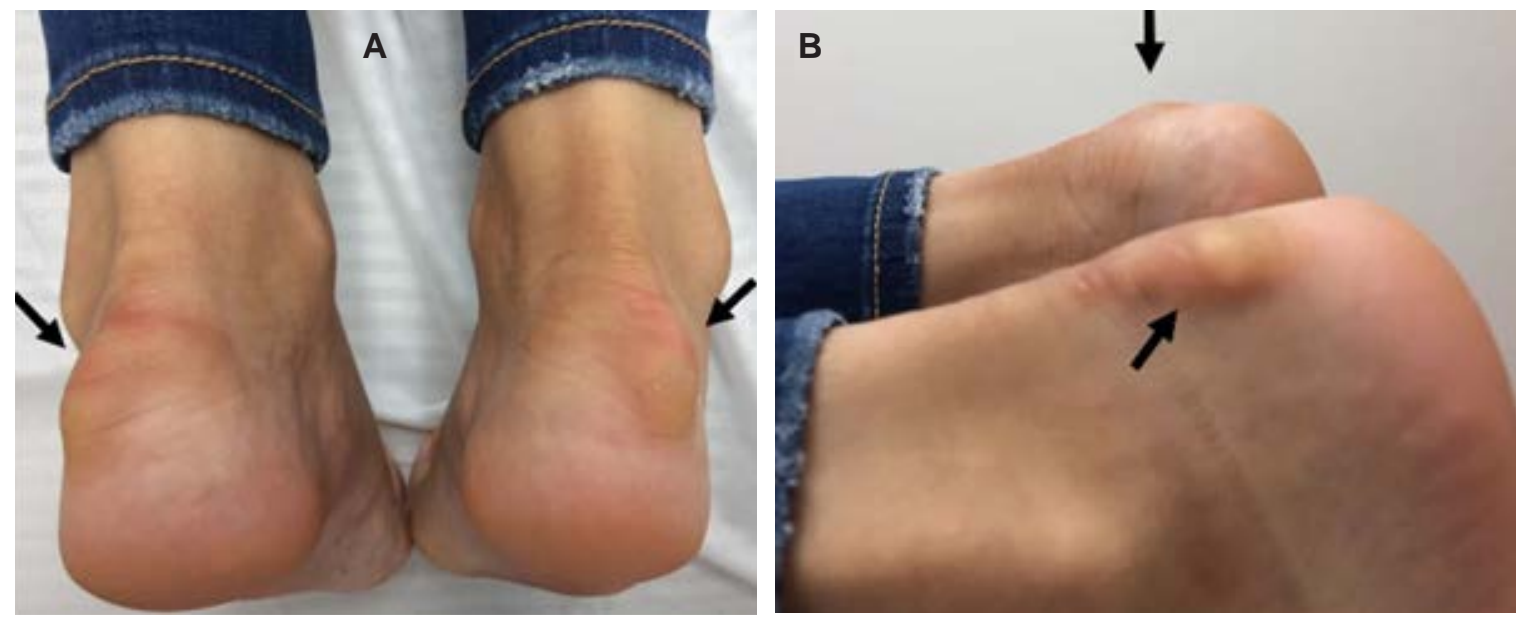

Figura 1: Fotografías clínicas de cara posterior de ambos talones. A) Vista posterior. B) Vista lateral que muestra engrosamiento de la inserción lateral del tendón de Aquiles y prominencia de la bursa retrocalcánea.

\footnotetext{
* Ortopedista. Cirugía Articular. División de Cirugía del Hospital Ángeles León, León, Guanajuato. México.

* Especialista en Medicina de Rehabilitación. Catedrático de la Facultad de Medicina de León, Universidad de Guanajuato. México.
}

\section{Correspondencia: \\ Dr. Luis Gerardo Domínguez Carrillo \\ Correo electrónico: Igdomínguez@hotmail.com \\ Aceptado: 02-08-2020.}

www.medigraphic.com/actamedica 


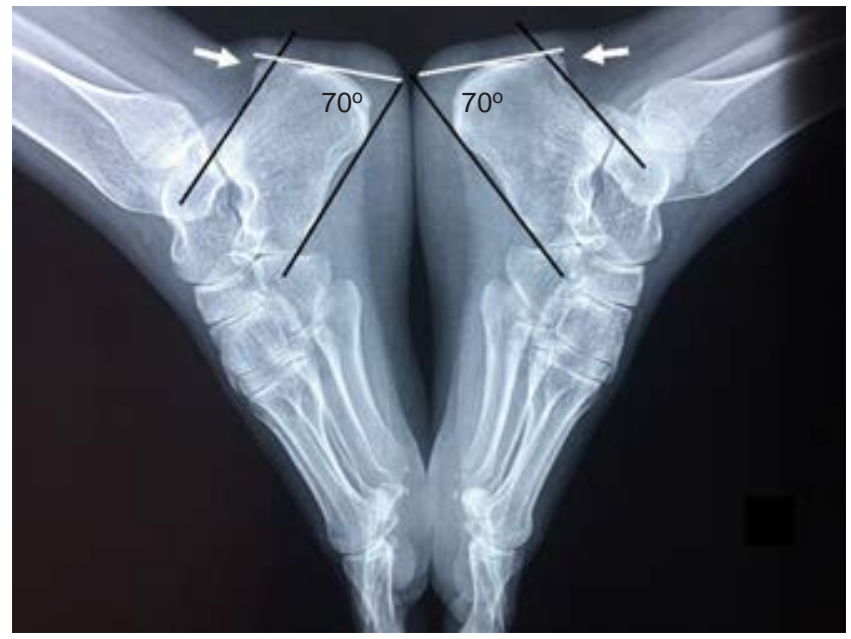

Figura 2: Radiografías laterales de ambos pies, el ángulo de Fowler (normal entre 44 y $70^{\circ}$ ) se define por la intersección de una línea tangencial a la prominencia posterosuperior de la proyección de la bolsa y otra línea tangencial al tubérculo anterior y la tuberosidad medial. Las líneas paralelas se definen por la tangente basal al tubérculo anterior y la tuberosidad medial y una línea paralela desde el labio posterior de la faceta articular talar. Cuando la tuberosidad posterosuperior supera la línea superior se puede considerar deformidad de Haglund.

punto de corte 2.5, cuando la relación es menor de 2.5 corresponde a deformidad de Haglund. ${ }^{3}$

En el diagnóstico diferencial se debe incluir la xantomatosis del tendón de Aquiles, algunas entesitis (como el síndrome de Reiter) y las artritis gotosa y reumatoide, que pueden provocar similar aumento de partes blandas.

En el manejo conservador con tratamiento médico, fisiátrico y ortésico se indica siempre el uso de calzado sin talón; de no mejorar en un lapso de seis meses, se plantea el tratamiento quirúrgico que consiste en el retiro

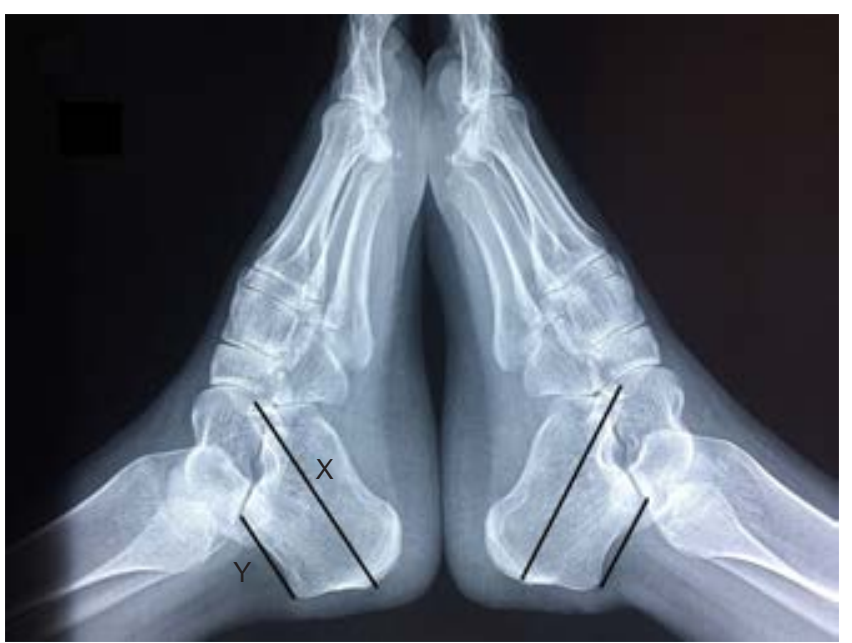

Figura 3: Radiografías laterales de ambos pies que muestran el trazado de las líneas para efectuar la relación calcáneo X/Y con un valor de corte de 2.5, cuando es menor de 2.5 corresponde a deformidad de Haglund, en este caso la relación correspondió la relación mostró 2.25 .

de la bursa retrocalcánea y la resección de la deformidad de Haglund. ${ }^{4}$

\section{REFERENCIAS}

1. Jiménez MF, Alonso VM, Díaz PG, Fernández LJ, Hernández HD, Díaz GF. Haglund's syndrome. Two cases report. Reumatol Clin. 2017; 13 (1): 37-38. doi: 10.1016/j.reuma.2015.12.006.

2. Bulstra GH, van Rheenen TA, Scholtes VA. Can we measure the heel bump? Radiographic evaluation of Haglund's deformity. J Foot Ankle Surg. 2015; 54 (3): 338-340.

3. Tourné $\mathrm{Y}$, Baray AL, Barthelemy R, Moroney P. Contribution of a new radiologic calcaneal measurement to the treatment decision tree in Haglund syndrome. Orthop Traumatol Surg Res. 2018; 104 (8): 1215-1219. doi: org/10.1016/j.otsr.2018. 08.014.

4. Gianni RA, Stewart M, Thompson D, Collier WB, Ashmyan R. The central-splitting approach for Achilles insertional tendinopathy and Haglund deformity. JBJS Essent Surg Tech. 2020; 10: e0035. doi: 10.2106/JBJS.ST.19.00035. 